\title{
A wide field-of-view low-resolution spectrometer at APEX: Instrument design and scientific forecast
}

The CONCERTO Collaboration: P. Ade ${ }^{1}$, M. Aravena ${ }^{2}$, E. Barria ${ }^{3,4}$, A. Beelen ${ }^{5}$, A. Benoit ${ }^{3,4}$, M. Béthermin ${ }^{5}$,

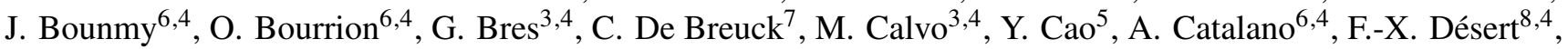

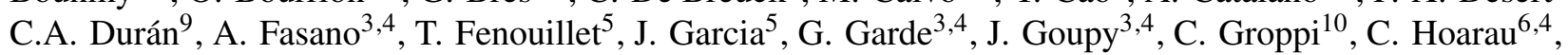
G. Lagache ${ }^{5}$, J.-C. Lambert ${ }^{5}$, J.-P. Leggeri ${ }^{3,4}$, F. Levy-Bertrand ${ }^{3,4}$, J. Macías-Pérez ${ }^{6,4}$, H. Mani ${ }^{10}$, J. Marpaud ${ }^{6,4}$, P. Mauskopf ${ }^{10}$, A. Monfardini ${ }^{3,4}$, G. Pisano ${ }^{1}$, N. Ponthieu ${ }^{8,4}$, L. Prieur ${ }^{5}$, S. Roni ${ }^{6}$, S. Roudier $^{6}$, D. Tourres ${ }^{6,4}$, and C. Tucker ${ }^{1}$

\footnotetext{
1 Astronomy Instrumentation Group, University of Cardiff, The Parade CF24 3AA, UK

2 Núcleo de Astronomía, Facultad de Ingeniería y Ciencias, Universidad Diego Portales, Av. Ejército 441, Santiago, Chile

${ }^{3}$ Univ. Grenoble Alpes, CNRS, Grenoble INP, Institut Néel, 38000 Grenoble, France e-mail: monfardini@neel.cnrs.fr

4 Groupement d'Interet Scientifique KID, 38000 Grenoble and 38400 Saint Martin d'Hères, France

5 Aix Marseille Université, CNRS, LAM (Laboratoire d'Astrophysique de Marseille), 13388 Marseille, France e-mail: guilaine. lagache@lam.fr

${ }^{6}$ Univ. Grenoble Alpes, CNRS, LPSC/IN2P3, 38000 Grenoble, France

7 European Southern Observatory, Karl Schwarzschild Straße 2, 85748 Garching, Germany

8 Univ. Grenoble Alpes, CNRS, IPAG, 38400 Saint Martin d'Hères, France

9 European Southern Observatory, Alonso de Cordova 3107, Vitacura, Santiago, Chile

10 School of Earth and Space Exploration and Department of Physics, Arizona State University, Tempe, AZ 85287, USA
}

Received 20 May 2020 / Accepted 24 July 2020

\begin{abstract}
Context. Characterising the large-scale structure in the Universe from present times to the high redshift epoch of reionisation is essential to constraining the cosmology, the history of star formation, and reionisation, to measuring the gas content of the Universe, and to obtaining a better understanding of the physical processes that drive galaxy formation and evolution. Using the integrated emission from unresolved galaxies or gas clouds, line intensity mapping (LIM) provides a new observational window to measure the larger properties of structures. This very promising technique motivates the community to plan for LIM experiments.

Aims. We describe the development of a large field-of-view instrument, named CONCERTO (for CarbON CII line in post-rEionisation and ReionisaTiOn epoch), operating in the range 130-310 GHz from the APEX 12-m telescope (5100 m above sea level). CONCERTO is a low-resolution spectrometer based on the lumped element kinetic inductance detectors (LEKID) technology. Spectra are obtained using a fast Fourier transform spectrometer (FTS), coupled to a dilution cryostat with a base temperature of 0.1 K. Two two kilo-pixel arrays of LEKID are mounted inside the cryostat that also contains the cold optics and the front-end electronics.

Methods. We present, in detail, the technological choices leading to the instrumental concept, together with the design and fabrication of the instrument and preliminary laboratory tests on the detectors. We also give our best estimates for CONCERTO sensitivity and give predictions for two of the main scientific goals of CONCERTO, that is, a [CII]-intensity mapping survey and observations of galaxy clusters.

Results. We provide a detailed description of the instrument design. Based on realistic comparisons with existing instruments developed by our group (NIKA, NIKA2, and KISS), and on the laboratory characterisation of our detectors, we provide an estimate for CONCERTO sensitivity on the sky. Finally, we describe, in detail, two of the main scientific goals offered by CONCERTO at APEX.
\end{abstract}

Key words. instrumentation: detectors - instrumentation: spectrographs - telescopes - cosmology: observations

\section{Introduction}

Modern imaging and polarimetry cameras, at millimetre and sub-millimetre wavelengths, are currently operating on large (e.g. $D>10 \mathrm{~m}$ ) single-dish telescopes. The main goal of these instruments is to map, at relatively high angular resolution (e.g. 5-30 arcsec), large portions of the sky (e.g. several $\mathrm{deg}^{2}$ ) with a high sensitivity (e.g. $\mathrm{RMS}_{\mathrm{MAP}} \lesssim 1 \mathrm{mJy}$ ). Polarised emissions are also measured with similar specifications.

In this context, the dual-band NIKA2 camera represents the first kilo-pixel instrument operating at these wavelengths based on the kinetic inductance detectors (KID) technology (Adam et al. 2018; Perotto et al. 2020). The particular type of KID used for NIKA2 are front-illuminated lumped element KID (LEKID; Doyle et al. 2010). They consist in inductor-capacitor (LC) superconducting planar resonators made by a long meandered inductor (wire), which is terminated at both ends by an interdigitated capacitor. NIKA2 supersedes previous cameras based on transition edge sensors (referred to as TES bolometers) in the frequency range of $150-360 \mathrm{GHz}$, such as MAMBO2 at IRAM (Kreysa et al. 1998), LABOCA at APEX (Siringo et al. 2009), and SCUBA-2 at the JCMT (Holland et al. 2013).

In order to extend the capabilities of the existing instruments and to open new observational windows of the millimetre 
sky, the spectral dimension has to be added, without sacrificing the instantaneous field-of-view. The large field-of-view and the mapping speed are, actually, the main asset of single dish telescopes when compared to variable baseline interferometers, such as ALMA $^{1}$ or NOEMA (Lefèvre et al. 2020). For this reason, we are developing a millimetre-wave low spectral resolution $(R=$ $v / \Delta v \leq 300)$ spectrometer with an instantaneous field-of-view of $20 \mathrm{arcmin}$. In order to preserve the angular resolution at frequencies of around $300 \mathrm{GHz}$ and assuming a 10-m class telescope, a focal-plane containing around 2000 spatial pixels is needed. Toachieve these figures, we adopt a room-temperature MartinPuplett Interferometer (MpI) (Martin \& Puplett 1970) coupled to a large field millimetre-wave LEKID camera. The instrument, named CONCERTO (for CarbON CII line in post-rEionisation and ReionisaTiOn epoch), has been designed to interface with the Atacama Pathfinder EXperiment (APEX) 12-m telescope (Güsten et al. 2006). A pathfinder instrument, named KISS that is based on the same concept, has been built by our collaboration and deployed in November 2018, at the Teide Observatory (Fasano et al. 2020a).

One important scientific driver that has motivated our developments is the study of the [CII] emission line at high redshift. The $[\mathrm{CII}]$ line is among the brightest lines originating from starforming galaxies and it is a reliable tracer of star formation on global scales. With CONCERTO at APEX, we will map, in three dimensions, the fluctuations of the [CII] line intensity in the reionisation and post-reionisation epoch $(z \gtrsim 5)$. This technique, known as "intensity mapping", will allow us to answer the questions of whether dusty star-formation contributes to early galaxy evolution, and whether [CII]-emitters play an important role in shaping cosmic reionisation. The dedicated [CII] survey will provide a (spatial-spectral) data cube in which intensity is mapped as a function of the sky position and frequency. The 3D fluctuations will then be studied in Fourier space with the power spectrum. The [CII] survey will also be sensitive to the $\mathrm{CO}$ intensity fluctuations arising from $0.3<z<2$ galaxies, giving the spatial distribution and abundance of molecular gas over a broad range of cosmic time. The [CII] intensity mapping is also one of the main goals of CCAT-prime (Choi et al. 2020) and TIME (Crites et al. 2014), which are two experiments that are based on different technologies than CONCERTO: Gratings and TES bolometers are used for TIME, and KID and FabryPerot interferometers are used for CCAT-p. In addition to the main [CII] survey, we expect CONCERTO to bring a significant contribution in a number of areas, including the study of galaxy clusters (via the thermal and kinetic SZ effect), the observation of local and intermediate-redshift galaxies, and the study of Galactic star-forming clouds. In this paper, we detail the main goals of the $[\mathrm{CII}]$ intensity mapping survey and galaxy cluster observations.

The paper is organised as follows. We present the instrumental concept, design, and preliminary results in Sect. 2. The discussion pertains to KID detectors, cryogenics, and optics. In Sect. 3, we describe the first laboratory tests. In Sect. 4, we present the sensitivity estimates, while Sect. 5 is dedicated to the $[\mathrm{CII}]$ intensity mapping and SZ surveys.

\section{CONCERTO instrument}

CONCERTO has been specifically designed to fit into the Cassegrain cabin (C-cabin) of the APEX telescope. It is composed of two main components: the so-called chassis and the

\footnotetext{
1 https://almascience. eso.org
}

Table 1. Main characteristics of CONCERTO.

\begin{tabular}{lc}
\hline \hline Telescope primary mirror diameter [m] & 12 \\
Field-of-view diameter [arcmin] & 20 \\
Absolute spectral resolution [GHz] & $\geq 1$ \\
Relative spectral resolution $R$ [\#] & $1-300$ \\
Frequency range HF | LF [GHz] & $195-310 \mid 130-270$ \\
Pixels on Sky HF | LF [\#] & $2152 \mid 2152$ \\
Angular resolution HF | LF [arcsec] & $20-32 \mid 23-45$ \\
Average angular resolution HF | LF [arcsec] & $26 \mid 34$ \\
Instrument geometrical throughput [sr m $\left.{ }^{2}\right]$ & $2.5 \times 10^{-3}$ \\
Single Pixel geometrical throughput $\left[\mathrm{sr} \mathrm{m}^{2}\right]$ & $1.16 \times 10^{-6}$ \\
Data rate [MBytes s ${ }^{-1}$ ] & 128 \\
\hline
\end{tabular}

Notes. For comparison, the NIKA2 instrument (6.5' field-of-view, 30metre telescope) geometrical throughput $(\mathrm{A} \times \Omega)$, characterising the camera collecting power, is $1.7 \times 10^{-3} \mathrm{sr} \mathrm{m}^{2}$. Concerning the overall optical transmission of CONCERTO compared to NIKA2, we refer the reader to Sect. 4.1.

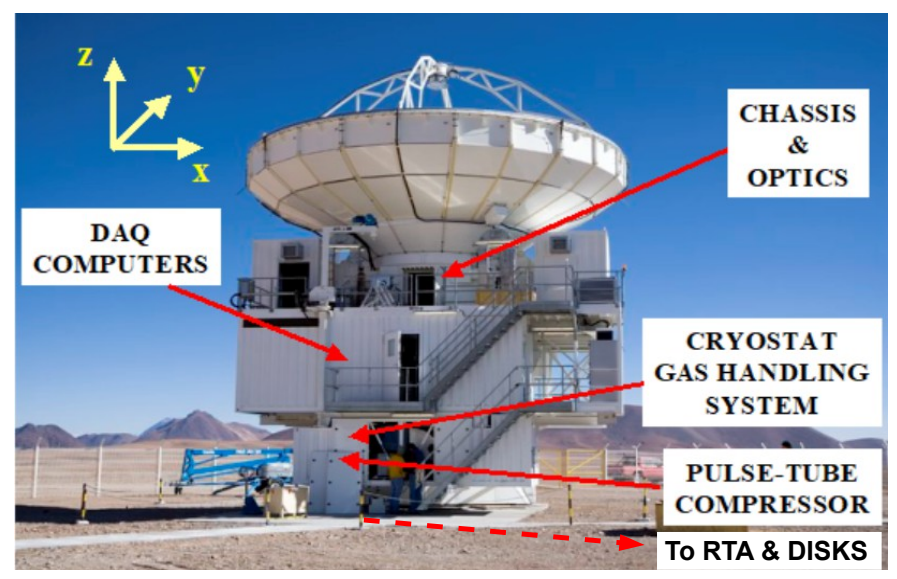

Fig. 1. Location of the CONCERTO sub-systems: the chassis and optics in the C-cabin, the Data AcQuisition computers (DAQ) in the middle container ("instrumentation container"), and the gas handling system and the pulse-tube compressor in the bottom container ("compressors room"). Real time analysis (RTA) computers and hard disks are not in the telescope tower.

optics box. The "chassis" includes the camera (cryostat), the MpI interferometer, the readout, and control electronics. The "optics box" includes a number of mirrors and polarisers as well as a cold reference for the MpI. In Table 1 we summarise the main instrument characteristics. The location of the CONCERTO subsystems is shown in Fig. 1.

In this section, we describe, in detail, the camera and its content (Sect. 2.1), the MpI (Sect. 2.2), and the chassis and related electronics (Sect. 2.3). The room temperature optics, including the cold reference source, is presented in Sect. 2.4. Section 2.5 is devoted to a brief description of the CONCERTO hardware components, which are located elsewhere than in the telescope tower. Information about the installation at the telescope is found in Sect. 2.6.

\subsection{The camera}

The CONCERTO camera is based on a cryogenic-liquid-free custom dilution cryostat. The dilution insert and the pulsetube orientation, in particular, have been specifically designed to allow the rotation of the cryostat axis following telescope 


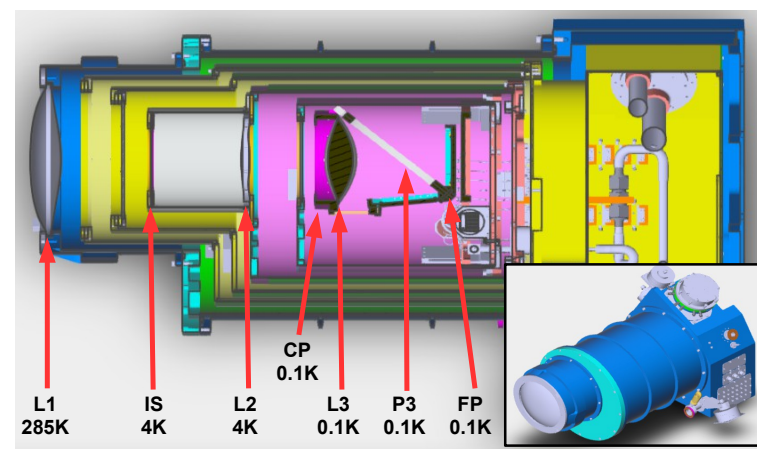

Fig. 2. CONCERTO camera cross-section and 3D view (inset). The positions of the three HDPE lenses are shown (L1, L2, L3), together with the image stop (IS), the cold pupil (CP), the cold polariser (P3), and the two focal planes (FP). The diameter of L1 is around $250 \mathrm{~mm}$.

movements. The cryostat is optimised for the range of telescope elevations (EL) comprised between 30 and 90 degrees. The best working point is achieved for $E L=60$ degrees.

The main camera optical features, shown in Fig. 2, are the image stop (IS) at a temperature of $4 \mathrm{~K}$ and the cold pupil (CP) at the base temperature of around $0.1 \mathrm{~K}$. Three HDPE (High Density PolyEthilene) lenses are used in the camera: L1 (room temperature), L2 $(4 \mathrm{~K})$, and L3 $(0.1 \mathrm{~K})$. In order to analyse the polarised signal, the last polariser of the $\mathrm{MpI}(\mathrm{P} 3)$ is placed just in front of the LEKID arrays (FP) at the base temperature. P3 is a custom wire-grid polariser. It is realised on a $12 \mu \mathrm{m}$-thick Polyimide membrane, and with Copper wires with a pitch of $50 \mu \mathrm{m}$. A number of infrared (IR)-blocking (thermal) and metallic multi-mesh filters (Ade et al. 2006) are mounted at different stages. In particular, we have thermal filters on the warmest stages (room temperature to $50 \mathrm{~K}$ ), low-pass multi-mesh filters at the intermediate temperatures $(50 \mathrm{~K}-1 \mathrm{~K})$, and band-defining filters at the base temperature (i.e. just in front of each focal-plane array). A specially blackened baffle is installed at $4 \mathrm{~K}$, between IS and L2, in order to suppress the stray light.

Since LEKID are sensitive to variations of magnetic fields, they have to be protected by a multi-stage B field screen. Four concentric high-permittivity alloy (i.e. mu-metal and cryogenics variations) cylinders are installed at $300 \mathrm{~K}, 50 \mathrm{~K}$ (double screen), and $4 \mathrm{~K}$. An additional superconducting screen will be wrapped around the focal planes section.

The focal plane arrays are microstrip-coupled LEKID similar to those used for NIKA2 (Adam et al. 2018). Six feed-lines (i.e. excitation and readout lines) are needed to read out each of the 2152 pixel arrays. A total of twelve pairs of coaxial cables are thus running into the cryostat. The pixels design itself is derived from NIKA (Monfardini et al. 2011). The LEKID details have been optimised to meet the CONCERTO specifications. In particular, the shape of the meander and its coupling quality factor have been adjusted to the target range of frequencies and expected background. The coupling quality factor is designed to be $Q_{\mathrm{c}} \approx \Delta f_{-3 \mathrm{~dB}} / f_{0} \approx 2.5 \times 10^{4}$, where $\Delta f_{-3 \mathrm{~dB}}$ is the typical width of the resonance under dark conditions. The thickness of the dielectric substrate is calibrated in order to maximise the quantum efficiency, and it is in the range of 100-120 $\mu \mathrm{m}$.

The fabrication process (Goupy et al. 2016) looks straightforward when compared to competing detectors that have similar performances. The substrate, a 100-millimetre high-purity mono-crystalline silicon wafer $^{2}$, is prepared in the deposition

\footnotetext{
https://www.sil-tronix-st.com/en/
}

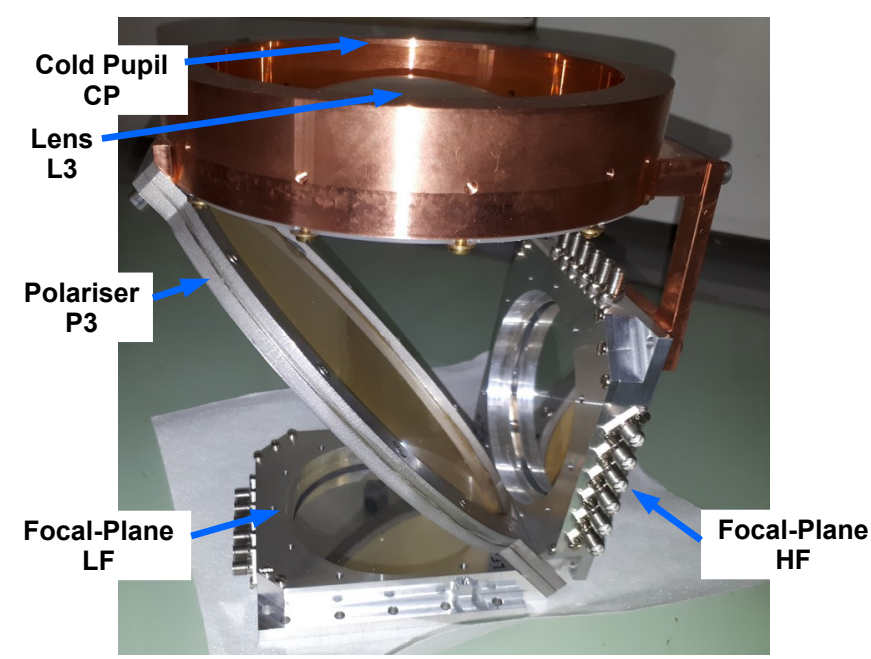

Fig. 3. Picture of the first " $100 \mathrm{mK}$ block" including the cold pupil, the L3 lens, the P3 polariser, and the two arrays (HF and LF) containing 2152 pixels each. The array holders are, in this case, realised in Aluminium. A version of the block with Copper holders is also available.

chamber by soft ion milling. The superconducting film deposition, which is made of Aluminium with a thickness of $20 \mathrm{~nm}$, is achieved by e-beam evaporation and under a residual chamber pressure of $5 \times 10^{-8}$ mbars. The deposition rates is fixed at $0.1 \mathrm{~nm} \mathrm{~s}^{-1}$. The ultraviolet (UV) photo-lithography step is based on a positive resist and is followed by wet etching. The etching is done using a standard aluminium etching solution based on phosphoric acid. The diced detectors arrays are packaged in custom holders and bonded, via $17 \mu \mathrm{m}$ Aluminium wires, to the 50-Ohms micro-strip launchers. Those are then tin-soldered to the inner pin of the $\mathrm{SMA}^{3}$ feed-throughs.

The front-end electronics stage is installed in the cryostat, at a temperature of $4 \mathrm{~K}$. It is made of a series of twelve lownoise amplifiers (LNA) operating at the resonance frequencies ${ }^{4}$, that is, in the range of $1.5-2.5 \mathrm{GHz}$. A second stage of cryogenic amplification, made of twelve commercial LNA, has been added on the $50 \mathrm{~K}$ cryogenic stage to simplify the room-temperature electronics and reduce its power consumption. The connections between the cold electronics stages, the arrays, and the SMA vacuum feed-throughs plate (see Fig. 2), are ensured by commercial semi-rigid cryogenic coaxial cables. In particular, we adopt NbTi superconducting coaxial cables for the portion connecting the output of the LEKID arrays to the input of the front-end amplifiers. Fixed attenuators are mounted on each input line at the $4 \mathrm{~K}$ stage. The overall electrical gain of each radio-frequency line to and from the room temperature electronics has been measured and is about $+25 \mathrm{~dB}$.

\subsection{The Martin-Puplett interferometer}

The MpI is a particular kind of Fourier transform spectrometer (FTS). It is capable of measuring the differential spectrum of a source, with respect to a given reference. The key elements are three polarisers (P1-beam divider, P2-splitter, and P3-analyser), two (one fixed and one variable) arms, and two (one fixed and one moving) rooftop mirrors (see Fig. 4). This technique is widely adopted in the millimetre and sub-millimetre domains,

\footnotetext{
3 SubMiniature version A.

4 http://thz.asu.edu/products.html
} 


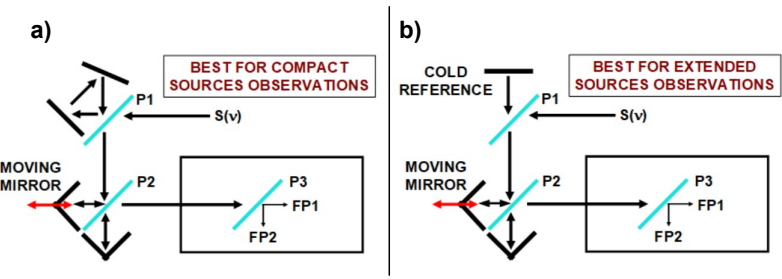

Fig. 4. Schematics of the MpI concept. Two options are shown for the reference source: $(a)$ a de-focused image of the instantaneous field-ofview; and $(b)$ a cold reference. The polariser P1 provides the needed polarised input to the MpI. P2 is the beam splitter defining the two arms, while $\mathrm{P} 3$, in the cryostat, dispatches the two projections of the polarised signal to the focal-plane arrays. The incoming beam is represented by a spectral distribution $S(v)$.

mostly for laboratory characterisations, but also for narrow ${ }^{5}$ field-of-view observations from space in the past (Mather 1999; Griffin et al. 2010). The first examples of wide field instruments making use of an MpI to obtain spectral information are the stratospheric balloon OLIMPO (Schillaci et al. 2014) and the previously mentioned KISS ground-based spectro-photometer (Fasano et al. 2010b).

To achieve a spectral resolution better than $1 \mathrm{GHz}$, the maximum range $\Delta l_{\max }$ of the moving rooftop mirror has to be larger than $75 \mathrm{~mm}$. In CONCERTO, the motors can move by up to $90 \mathrm{~mm}$. The range $\Delta \mathrm{l}$ spanned by the interferogram can be adjusted, on a scan-by-scan basis and depending on the science target, from zero to the maximum. The spectral resolution will then be

$\Delta v=\frac{c}{4 \times \Delta l}$.

The optical path difference is thus $\mathrm{OPD}=2 \Delta l$. Leaving the rooftop mirror immobile at the zero-path difference position results in using CONCERTO as a broad- and dual-band large field-of-view imager.

The distinctive feature of the CONCERTO (and KISS) MpI is the combination of the speed of movement of the rooftop mirror and its size and mass. In order to avoid atmospheric drifts during a single interferogram, the mechanical frequency of the motors is set to around $4 \mathrm{~Hz}$, that is, eight full interferograms (and spectra) per second are produced by each of the pixels. The lateral size of the mirror that is to be moved exceeds $0.5 \mathrm{~m}$ for a mass exceeding $3 \mathrm{~kg}$. In order to counterbalance the linear momentum associated with this moving mass, a second motor, with an equivalent mass, oscillates with an opposite instantaneous velocity. With a maximum force around $1000 \mathrm{~N}$, the acceleration that can be imposed to the moving mass, including the motor piston, exceeds $100 \mathrm{~m} \mathrm{~s}^{-2}$. The theoretical curve that is commanded to the motor is a square wave (i.e. a constant speed for both directions and a maximum acceleration at the turnbacks). The real curve is of course smoothed out by the finite acceleration near the extremes. We present a picture of the system that was built for CONCERTO in Fig. 5.

\subsection{The chassis and electronics}

The chassis is a single, compact support structure to which many of the core components of CONCERTO are attached. These

\footnotetext{
5 In this context, the field-of-view has to be expressed in the number of beams. The number of beams is around 2000 for CONCERTO and 300 for KISS, which can be compared to few tens, at most, for the previous instruments.
}

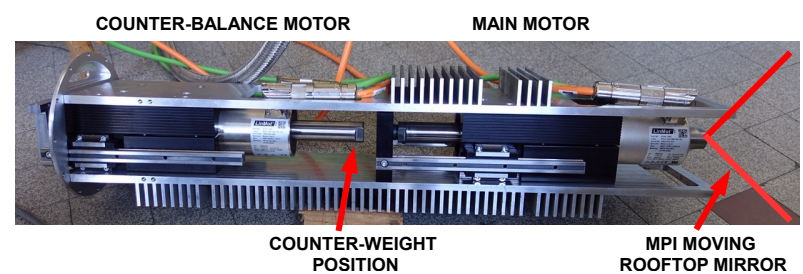

Fig. 5. CONCERTO double-motors MpI. Two identical linear motors, which develop a force of $\geq 1000 \mathrm{~N}$ each, are acted on in counter-phase to null the total momentum.

include the camera itself, the MpI motors and moving mirror, and the electronics boards, along with a large number of modules devoted to monitoring and controlling the instrument. The chassis was designed and fabricated to match the constraints related to the limited space available in the $\mathrm{C}$-cabin. It allows for the installation of multiple sub-systems of CONCERTO as a single element inside the APEX C-cabin. The chassis is pre-mounted in the laboratory and it can slide through the $\mathrm{C}$-cabin door.

Five microTCA ${ }^{6}$ racks mounted on the side of the chassis host the 12 advanced mezzanine cards (AMC) used to read out the two arrays. The cards have an architecture similar to those used for NIKA2 (Bourrion et al. 2012), but they have been improved to be able to generate up to 400 excitation tones spanning $1 \mathrm{GHz}$ in bandwidth. The data acquisition rate has been increased from less than $100 \mathrm{~Hz}$ to up to $4 \mathrm{kHz}$ in order to properly sample the interferograms generated by the MpI. The calibration strategy has been inherited from the NIKA and NIKA2 instruments (Calvo et al. 2014), but the continuous frequency modulation used there is no longer viable because of the highsampling rate. As a consequence, in CONCERTO, the position and shape of each resonance circle, which is used to calibrate the data, is reconstructed by sampling three points around the resonance: $f_{0}, f_{0}+\delta f$, and $f_{0}-\delta f$. The $\delta f$ is much smaller than the resonance width, and it is of the order of a few $\mathrm{kHz}$. The calibration step is performed at the beginning of each interferogram, while the direction of motion of the MpI rooftop mirror is changing (Fasano et al. 2020a). In our opinion, this approach represents the best trade-off between optimal calibration and observing efficiency, that is, the fraction of time that is devoted to the science data stream.

The moving elements inside the chassis, in particular the MpI motors and the gas flowing in the pulse-tube head, generate vibrations which could affect the detector performances. In order to suppress their propagation to the focal plane, the camera is fixed to the chassis only via a series of soft rubber pneumatic actuators, which strongly dampen the vibrations. Furthermore, the pressure inside the actuators (eight in total, with different orientations, as shown in Fig. 6) is constantly adjusted by a dedicated software, so that the cryostat position and axis, which are monitored by means of linear position transducers, are kept constant independently of the telescope elevation. The cryostat position adjustment, requiring a few seconds to complete, is done automatically after each re-pointing and, upon request, between two subsequent observing blocks (scans). The position is, on the other hand, monitored in real time even during scans.

\subsection{The cabin optics and the cold reference (optics box)}

The first CONCERTO element along the optical axis, after the telescope mirrors (M1 and M2), is the M3 foldable mirror mounted on the chassis. With a diameter of $900 \mathrm{~mm}$, it is

6 https://en.wikipedia.org/wiki/MicroTCA 

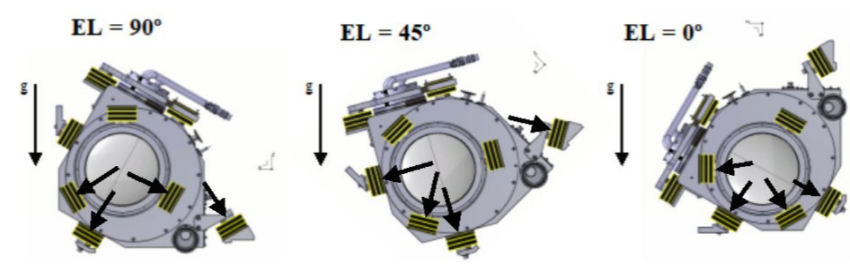

Fig. 6. Rotation of the camera (and chassis) following the telescope elevation (EL). The position of the eight soft rubber pneumatic actuators is shown. Two of them are dedicated to the pulse-tube head. For the six remaining actuators, we indicate (with black arrows) those that are in action for three representative elevation cases.

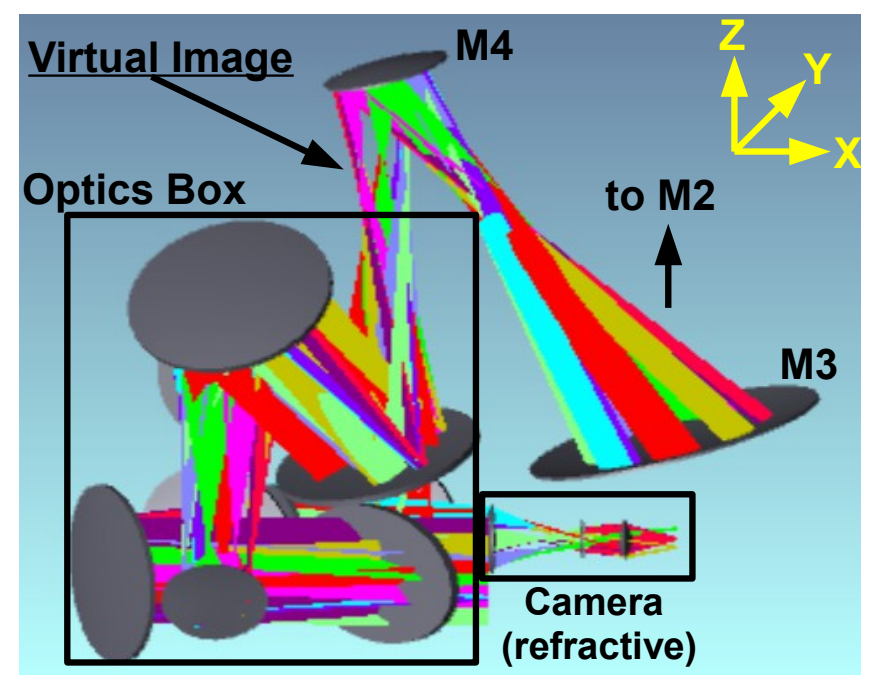

Fig. 7. 3D view of the CONCERTO optics, evidencing the M3 mirror interfaced to the APEX sub-reflector (M2). M4 is attached to the ceiling of the C-cabin and represents the only reflective optics component, with M3 and the MpI rooftop moving mirror, outside of the optics box. A large number of mirrors (M5 to M11), the two polarisers P1 and P2, and the cold reference optics complete the optical chain.

the largest among the CONCERTO mirrors. The next mirror, M4, which is attached to C-cabin upper ring, reflects the beam towards the $\mathrm{C}$-cabin floor and directly into the so-called optics box. A first virtual image is generated, by the combination M1M2-M3-M4, before M5. This virtual image plane will be used for some of the CONCERTO qualification tests.

The "optics box" includes a large number of mirrors (M5 to M11), the two polarisers P1 and P2 (Fig. 4) and the part of the optics providing the cold reference for the MpI. It also includes the fixed rooftop mirror of the interferometer. A general 3D view is shown in Fig. 7.

The mirrors are held at the C-cabin temperature, which is regulated at $11^{\circ} \mathrm{C}=284 \mathrm{~K}$. We expect an emissivity of the order of $1 \%$ per mirror, which is equivalent to an additional background of about $3 \mathrm{~K}$ per surface, so not smaller than $30 \mathrm{~K}$ in total. The stability of the temperature in the cabin is $\pm 1 \mathrm{~K}$. This means that, for an emissivity of $1 \%$, the effective background temperature variation induced per mirror is around $10 \mathrm{mK}$. Considering roughly ten mirrors, this translates to a total effective background temperature variation of $0.1 \mathrm{~K}$. The KID detectors, with a NET of the order of $\approx \mathrm{mK} \cdot \sqrt{s}$ per pixel, are sensitive to this drift that will produce a correlated signal on all of the pixels. However, these instabilities are harmless since they are slower and smaller than the atmospheric fluctuations sitting on top.

We performed a trade-off between the requirements related to the image quality (and the interferometry efficiency) and the

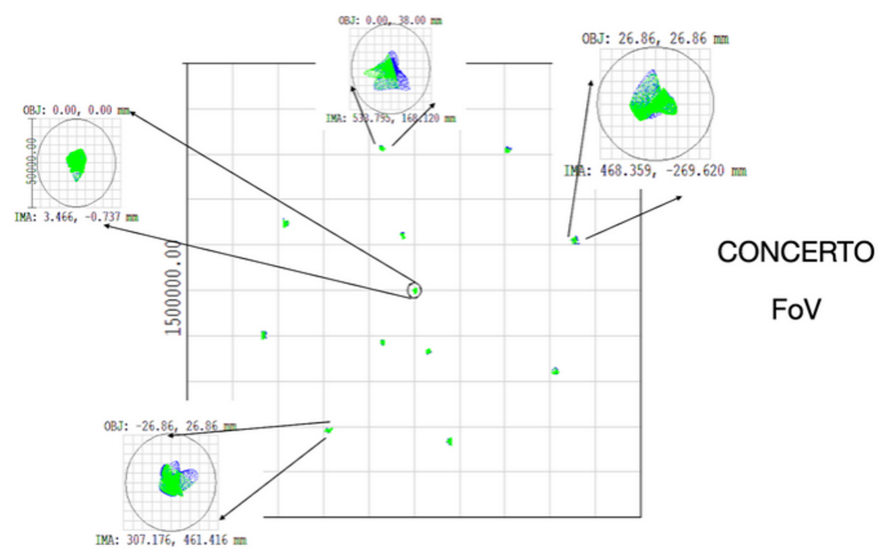

Fig. 8. Simulated focal plane image for the full, 20 arcmin, field-ofview. Each spot is shown for the two extreme positions of the roof mirror, i.e. green $(0 \mathrm{~mm})$ and blue $(90 \mathrm{~mm})$. The black circles represent the airy disks. It is clear that the "walking" of the beam is well contained in the diffraction disk.

lateral size of the MpI. In order to obtain a diffraction-limited combined beam for each position of the movable roof mirror (in the range of 0-90 $\mathrm{mm}$ ), we imposed the criterion, for each field on the sky, producing a quasi-parallel beam inside the MpI. According to the geometrical throughput conservation rule, the field-to-field divergence is thus fixed by the diameter of the beam. For the 20 arcmin field-of-view, and considering a $12 \mathrm{~m}$ primary mirror, we obtained an overall beam diameter of about $420 \mathrm{~mm}$ inside the interferometer. A consequence of this method is that, in requiring that the combined beam does not "walk" in the focal plane (see Fig. 8), we must accept a jitter on the entrance pupil of the optical system, that is, the "active" portion of the primary mirror. This is, in the end, the main reason why we have decided to under-sample the size of the illuminated primary mirror, to about $11 \mathrm{~m}$.

A remotely controllable three-position mirror is inserted in the optics chain in order to select the type of reference input for the MpI. The three options are as follows.

- The first option is sky, which is a de-focused image of the full 20 arcmin instantaneous field-of-view. The distinctive advantage is that the atmospheric common-mode spectrum is optically subtracted, providing a differential measurement of the astrophysics source spectrum with respect to the atmosphere along the line-of-sight. This means that, when targeting a field populated by weak sources, and at the first order, we obtain a null interferogram. This configuration is ideally suited for a compact object, that is, one with an angular extension smaller than 20 arcmin.

- The second option is an external cold black-body, which is a highly-emissive $(\epsilon \geq 0.98)$ cold disk that is cooled down by an independent pulse-tube cryostat $\left(T_{\mathrm{BB}} \approx 8 \mathrm{~K}\right)$. This configuration is mostly adapted to extended emission observations, that is, when the spectral and photometric gradients extend, on average, more than 20 arcmin.

- The third option is cold cryostat, which is a de-focused image of the CONCERTO cryostat cold (inner) parts. In other words, CONCERTO "looks" into itself to find a cold autoreference. Considering the number of optical elements (six mirrors and three lenses) lying between the three-position mirror and the coldest stage of the cryostat, we expect an equivalent effective temperature of the order of $20 \mathrm{~K}$. This is, in any case, lower than the loading of the sky plus the whole optics train between M1 and the focal plane, which is not lower than $50 \mathrm{~K}$. 


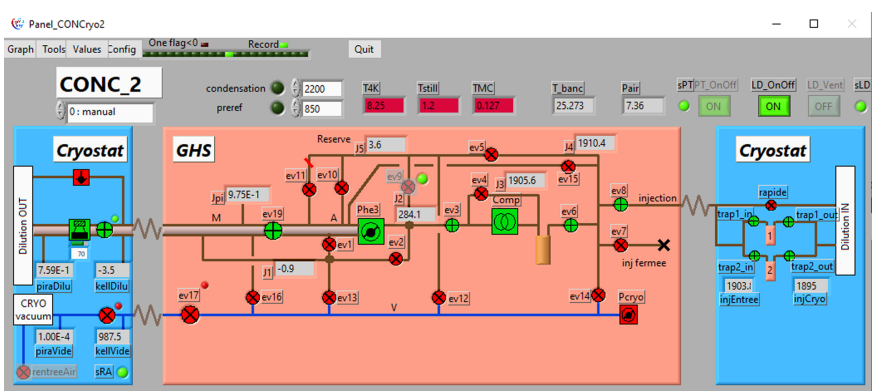

Fig. 9. GUI used to control the dilution refrigerator circuit.

This innovative configuration will be investigated as a simpler alternative to the external cold black body.

We stress the fact that the external cold black-body, or its alternative "old cryostat", are not used as spectral calibrators. They simply represent cold references, that is to say colder than the combined thermal emission of the atmosphere and the optical chain. In contrast to the "sky" reference case, the interferogram is not expected to be null at first order. In this case, we expect potential systematic effects to be minimised by the fact that both the reference and the dominant target (mostly the atmosphere in the common case of weak astrophysical sources) exhibit thermal black-body spectra.

The best choice between the three references will depend on the particular science target, the observing conditions and the unknown systematic effects as of yet affecting this new kind of large field-of-view spectrophotometer. A crucial phase of the onsky commissioning will be dedicated to investigating this item. We will report in further publications the results of this study, as well as a more detailed description of the CONCERTO MpI spectral reference system that is beyond the scope of the present paper.

\subsection{CONCERTO hardware outside the C-cabin}

In addition to the elements described above and located in the C-cabin, CONCERTO is also made by modules elsewhere in the telescope tower and beyond, in particular, the commercial pulsetube compressor (Cryomech CPA289C) and, more interestingly, the dilution cryostat gas handling system (GHS) and the Data AcQuisition (DAQ) and real time analysis (RTA) computers.

The GHS is composed of: (a) a series of pumps and compressors used to circulate the ${ }^{3} \mathrm{He}-{ }^{4} \mathrm{He}$ mixture and to provide compressed air to CONCERTO, and, (b) an electronic cabinet hosting a National Instrument CompactRIO ${ }^{7}$ real-time controller and multiple analogue and digital input-output modules. Dedicated Labview-based software is loaded on the CompactRIO. The software continuously monitors the state of the cryostat and controls all of the pumps, compressors, valves, and actuators of the dilution circuit. It can perform many tasks automatically, such as pre-cooling the system or putting it in a safe mode if the security thresholds are exceeded. It also acts as a server-side programme for the client graphical user interface (GUI) that is used on remote computers. The GUI allows one to easily see the state of the system and control its components (Fig. 9).

All of the pipes and cables that are needed to interconnect the elements of CONCERTO situated in different rooms (C-cabin, instrumentation, and compressors containers) are routed through flexible hoses. The hoses protect the CONCERTO cabling from

\footnotetext{
7 https://en.wikipedia.org/wiki/CompactRIO
}

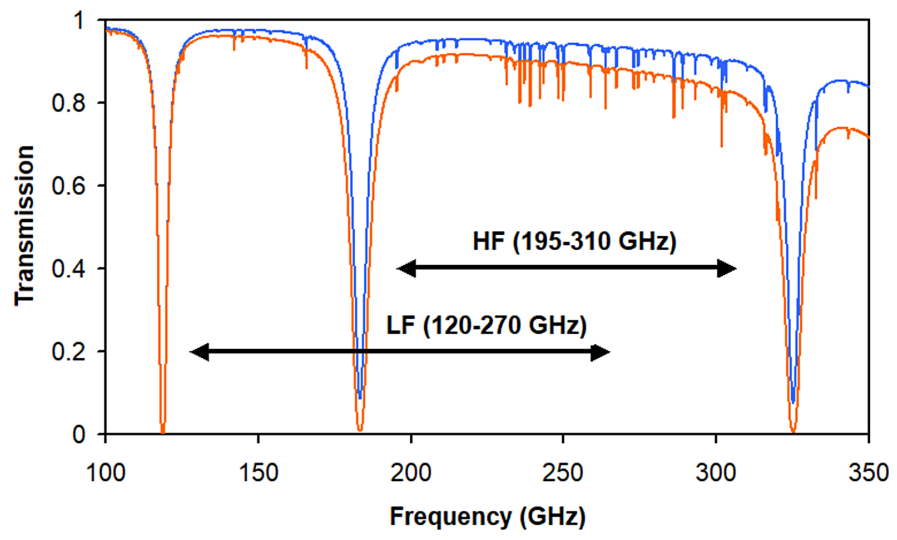

Fig. 10. Atmosphere transmission at APEX under $1 \mathrm{~mm}$ PWV (blue) and $2 \mathrm{~mm}$ PWV (orange) conditions. The CONCERTO bands for the $\mathrm{HF}$ and LF arrays are shown.

the environment and guarantee the flexibility required by the movement of the telescope. Inside the C-cabin, all of the connections are centralised on a dedicated panel located on the front side of the chassis, thus easing the procedures of plugging and unplugging.

The DAQ system is located in the middle container and is connected to CONCERTO in the $\mathrm{C}$-cabin, producing 128 MBytes per second, through five dedicated ethernet cables. The DAQ consists in two commercial computers with $48 \mathrm{~GB}$ of RAM and 24 cores each. The disk storage (432 TB) and the RTA systems are installed in the so-called servers room, located a few tens of metres away from the telescope tower. The RTA computer has 32 cores and 512 GB of RAM. The network connection between the DAQ and the disk storage or RTA is ensured by two 10 gigabit switches and underground cables.

\subsection{Installation at the APEX telescope}

The Atacama Pathfinder EXperiment (APEX) telescope is a modified prototype ALMA antenna with a primary mirror diameter of $12 \mathrm{~m}$ and a usable field-of-view of about 20 arcminutes. The location at around $5100 \mathrm{~m}$ a.s.l. on the Chajnantor plateau ensures optimal observing conditions (see Fig. 10). In particular, the fraction of time showing a precipitable water vapour (PWV) column that is lower than $2 \mathrm{~mm}$ is of the order of $70 \%$ or more.

The structure of the Cassegrain cabin, and in general the telescope infrastructure, had been designed to host large fieldof-view instrumentation. The primary mirror surface has been recently refurbished, and it achieves in some conditions a precision of the order of $10 \mu \mathrm{m}$ RMS. To date, APEX is thus a state-of-the-art installation for millimetre and sub-millimetre astronomy. Since 2007, the telescope has hosted, in the same place that will be occupied by CONCERTO, the Large APEX BOlometer CAmera (LABOCA) operating at $360 \mathrm{GHz}$ (Siringo et al. 2009).

The CONCERTO optics box and chassis are slid separately through the $\mathrm{C}$-cabin door and then fixed to the floor by a sufficient number of $12 \mathrm{~mm}$ metric screws. Since the beam will bounce between the floor and the top of the cabin (M4), we measured the deformations of the C-cabin itself under typical APEX observing conditions. This measurement was achieved using two linear wire sensors with a range of $3 \mathrm{~m}$ and a single measurement precision of $0.1 \mathrm{~mm}$. As is shown in Fig. 11, the root mean square (RMS) of both sensors, over 48 hours of data taken during 


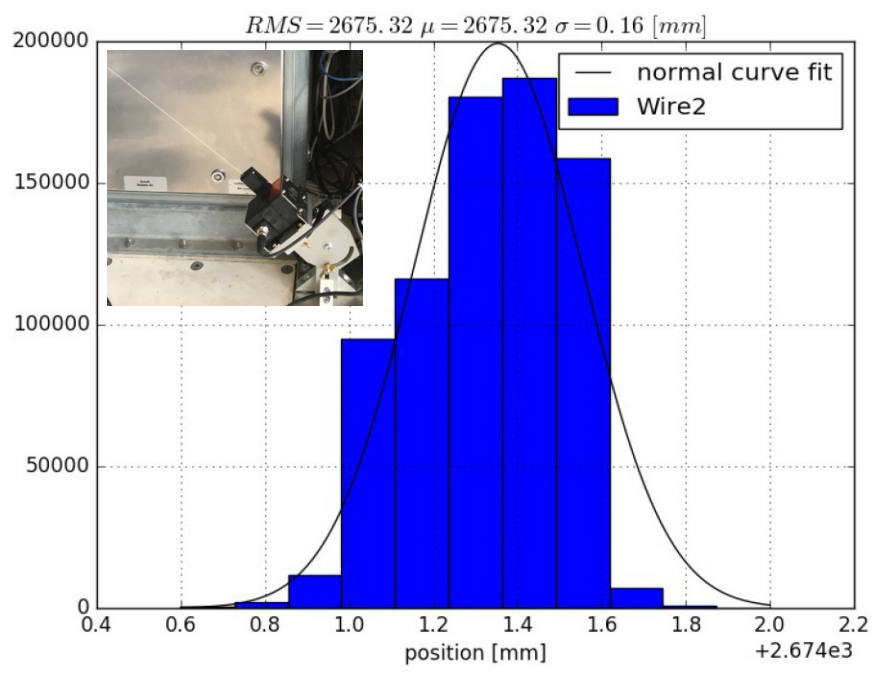

Fig. 11. Deformations of a wire sensor running between the floor and the top of the APEX Cassegrain cabin recorded for $48 \mathrm{~h}$. The average absolute length of the wire is $2675.3 \mathrm{~mm}$. Inset: picture of the wire sensor.

standard APEX observations, is smaller than $0.2 \mathrm{~mm}$ and thus negligible for our purposes.

The alignment of the mirrors in the optics box will be achieved in laboratory. The position of each mirror is adjusted with three micro-metric screws. No tuning will be possible at the telescope. The alignment of the optics box with respect to the chassis is ensured by the mechanical fixations. A set of specific lasers will be mounted to achieve the internal alignments in laboratory. At the telescope, we will use these lasers to align the optics box with respect to M4, M4 with respect to M3, and M3 with respect to M2. The alignment procedures will represent a critical step in the installation.

\section{Detectors laboratory characterisation}

In this section, we describe the first tests performed on CONCERTO detectors. Some of the electrical tests on the resonances occurred in the CONCERTO cryostat itself. On the other hand, the optical characterisation of the detectors has been achieved in the former NIKA2 test-bench. The so-called NIKA1.5 camera is an easily re-configurable optical dilution cryostat with a base temperature of $60 \mathrm{mK}$. It has been recently modified to host one CONCERTO array at a time. In particular, the optical filters can be easily replaced, and NIKA1.5 can be interfaced to a custom $\mathrm{MpI}$ for spectral characterisation, or alternatively to a sky simulator (described in detail in Monfardini et al. 2011) for sensitivity and beams' geometry measurements.

Since the twelve readout lines of the HF and LF arrays share a common local oscillator ( $\mathrm{LO}$, frequency reference for the readout electronics), it is of vital importance to accommodate all of the blocks of resonances in a common $\leq 1 \mathrm{GHz}$ band. This is nicely achieved, for example, in the case of the HF array shown in Fig. 12. The spread between blocks of resonances belonging to the same array, and between different arrays, is mainly due to inhomogeneities and uncertainties in the thickness of the Aluminium film.

Another important electrical parameter to be studied for large arrays of LEKID is the coupling quality factor $Q_{\mathrm{c}}$. The microstrip configuration that has been chosen has the advantage of guaranteeing a relatively well-peaked distribution of $Q_{\mathrm{c}}$. This

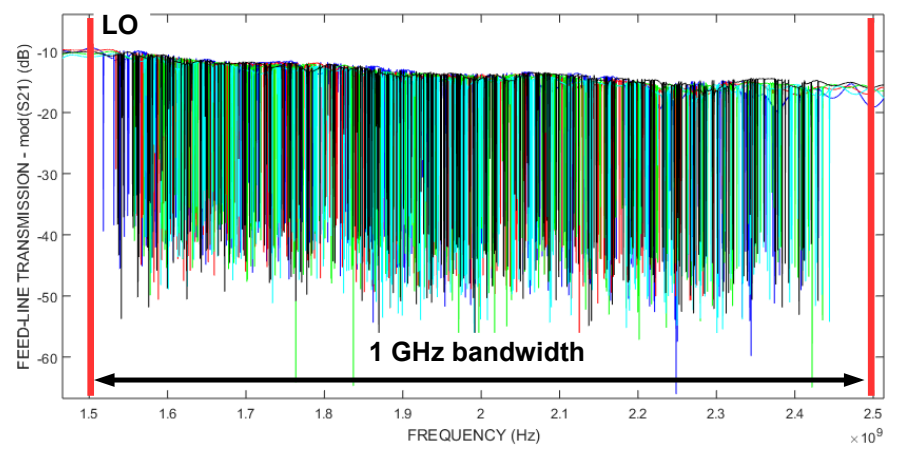

Fig. 12. Frequency sweep (transmission of the feed-line between port 1 and 2 , i.e. $\bmod (\mathrm{S} 21))$ of five blocks of resonances out of the baseline HF array. They have been acquired under dark conditions, in the CONCERTO cryostat, at $T=70 \mathrm{mK}$.

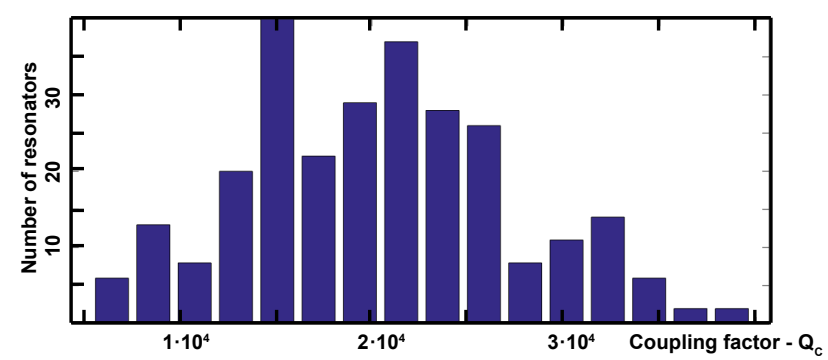

Fig. 13. Quality factors distribution for one representative block of resonances in the CONCERTO HF array. For this particular block $Q_{\mathrm{c}}=23 \mathrm{k} \pm 12 \mathrm{k}$, which is in line with all of the other blocks and with the designed $Q_{\mathrm{c}}=25 \mathrm{k}$. These quality factors were measured in the CONCERTO cryostat at $T=70 \mathrm{mK}$.

is achieved without requiring complicated and risky additional technology steps, such as cross-the-line micro-bondings or suspended micro-bridges. Figure 13 shows an example of the statistics obtained for one readout line of the CONCERTO HF array. All of the lines, as well as the LF arrays tested so far, exhibit similar behaviours. The quality factor distribution does, as designed, peak around $25 \mathrm{k}$.

The spectral response of two HF arrays, with slightly different substrate thicknesses, was measured. The results are reported in Fig. 14.

The sensitivity was measured in terms of NET (noise equivalent temperature), with the NIKA1.5 optics system. The average NET per pixel of the CONCERTO arrays, in NIKA1.5, is around $2 \mathrm{mK} \sqrt{\mathrm{Hz}^{-1}}$. This results in an NET of about $45 \mu \mathrm{K} \sqrt{\mathrm{Hz}^{-1}}$ per array (polarisation), or $32 \mu \mathrm{K} \sqrt{\mathrm{Hz}}^{-18}$ when combining both polarisations. Since the sensitivities per pixel are in accordance to what had been measured for the very similar NIKA2 detectors (Adam et al. 2018), we base our sensitivity estimate in Sect. 4 on NIKA2 values measured on-sky. We actually believe that the sensitivities measured on the maps on the sky for similar detectors are a more realistic prediction compared to somewhat ideal values estimated in laboratory.

The good imaging characteristics of the CONCERTO arrays are demonstrated by the Sky Simulator tests. An example is shown in Fig. 15. A deeper geometrical characterisation of the thousands of beams is beyond the scope of this paper and will only be performed on the final arrays.

8 We note that $32 \mu \mathrm{K} \sqrt{\mathrm{Hz}}^{-1}$ is equivalent to $22.6 \mu \mathrm{K} \mathrm{s}^{1 / 2}$. 


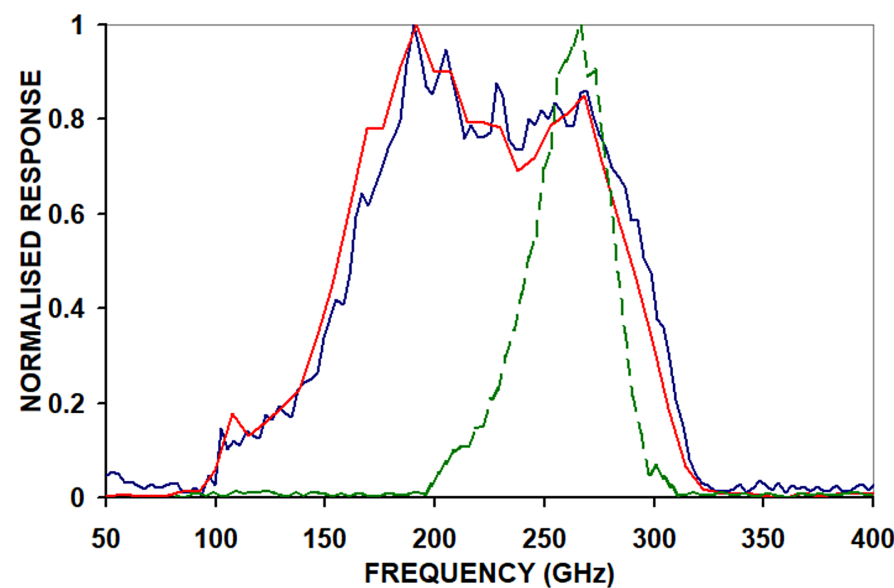

Fig. 14. Spectral response of two CONCERTO HF arrays (solid lines) and one NIKA2 $260 \mathrm{GHz}$ detector (dashed line). Red: HR Silicon substrate thickness of $110 \pm 5 \mu \mathrm{m}$; blue: thickness of $100 \pm 5 \mu \mathrm{m}$. Spectral responses were measured in the NIKA1.5 cryostat with low-pass filters defining an open band up to $300 \mathrm{GHz}$.

The typical response time of the LEKID used for CONCERTO ranges between $30 \mu \mathrm{s}$ and $100 \mu \mathrm{s}$, depending on the background. Even at the chosen sampling rate of $4 \mathrm{kHz}$, a cosmic-ray hit will thus represent a single-point glitch in the CONCERTO raw-time traces. The order of magnitude of the expected rate is $0.1 \mathrm{~Hz}$ per pixel.

\section{Sensitivity estimates}

Due to the similarities between the NIKA2 and CONCERTO detectors, we used the NIKA2 sensitivity measured on sky and on the reduced maps as a base to estimate the sensitivity for CONCERTO. As already advocated, we think that this approach, coupled with our NET laboratory measurements, provides quite realistic predictions.

\subsection{CONCERTO as an photometer: Dual-band sensitivity}

We first computed the sensitivity for CONCERTO as if it was a dual-band imager (LF and HF). For that, we relied on NIKA2 sensitivity measurements on the IRAM 30-m telescope. Aver-

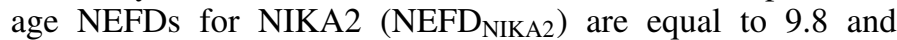
$36.1 \mathrm{mJy} \mathrm{s}^{1 / 2}$, at 150 and $260 \mathrm{GHz}$, respectively, for $\mathrm{pwv}=2$ and an elevation of 60 degrees (Perotto et al. 2020). These numbers already suffer from the transmission of the whole experiment.

We observe a large difference between the NIKA2 260 and $150 \mathrm{GHz}$ channel performances. A combination of known effects explains the gap in sensitivity. Indeed, at $260 \mathrm{GHz}$, (i) the beam efficiency of the 30-m telescope is about $55 \%$, (ii) the sensitivity is strongly affected (by $35 \%$ ) by a known defect of the NIKA2 dichroïc, and (iii) the contribution of residual sky noise to the average NEFDs is important. Therefore, as a realistic starting point for CONCERTO, we assume that the sensitivity for the LF array equals that of NIKA2 at $150 \mathrm{GHz}$. For the HF array, we assume a sensitivity better than that of the NIKA2 $260 \mathrm{GHz}$ channel, thanks to a gain in beam efficiency at APEX (which is of the order of $80 \%$ at the CONCERTO wavelengths), the lack of dichroïc in CONCERTO, and better atmospheric conditions. The values are thus (for $\mathrm{pwv}=2$ and an elevation of 60 degrees):

$\mathrm{NEFD}_{\mathrm{NIKA} 2}^{\mathrm{LF}}=10[7.5-15] \mathrm{mJy} \mathrm{s}^{1 / 2}$ and

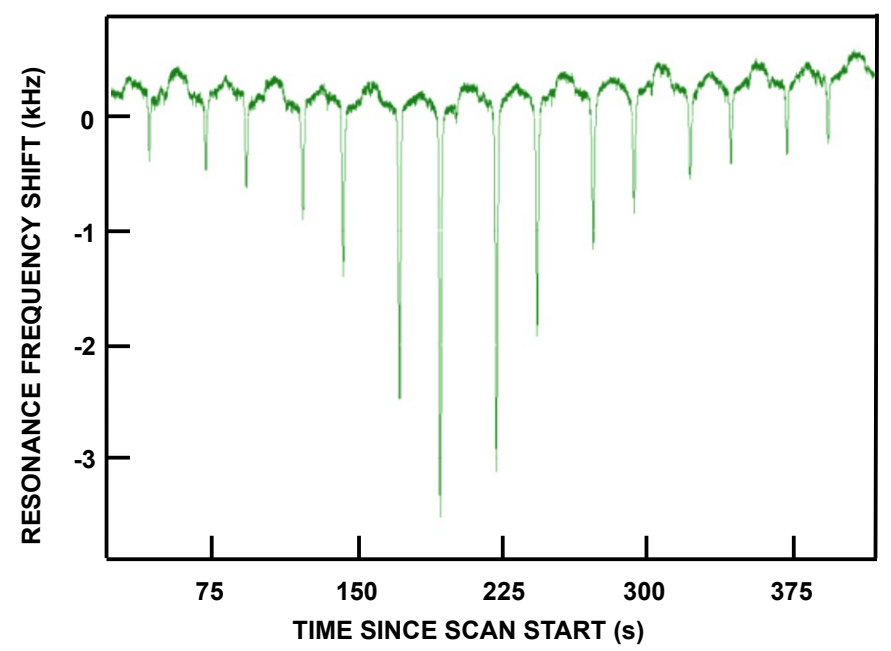

Fig. 15. Sky simulator trace. A fake "planet" (point-like source) is crossing the field-of-view of the considered pixel. A raster scan with subscans at a fixed elevation is simulated. The "elevation" steps are $4 \mathrm{~mm}$ long each. This measurement was obtained using the NIKA1.5 cryostat at $T=120 \mathrm{mK}$ and under a background temperature around $50 \mathrm{~K}$.

$\mathrm{NEFD}_{\mathrm{NIKA} 2}^{\mathrm{HF}}=15[10-20] \mathrm{mJy} \mathrm{s}^{1 / 2}$.

The numbers in brackets give the uncertainties on our assumption. As it was the case for NIKA2, we make the hypothesis that no excess noise will appear in CONCERTO at APEX compared to CONCERTO in laboratory. Of course, this cannot be verified until installation.

Compared to NIKA2, for CONCERTO, we have to scale the sensitivities to match the APEX telescope size and add two polarisers in the optical path (P1 and P2, see Fig. 4). Sensitivity loss is only due to $\mathrm{P} 1$, by a factor between $\sqrt{2}$ (if photon noise dominates) and 2 . To be conservative, we considered a factor of 2. Thus, for a single array of CONCERTO, the NEFD becomes:

$\mathrm{NEFD}^{\mathrm{LF}, \mathrm{HF}}=\mathrm{NEFD}_{\mathrm{NIKA} 2}^{\mathrm{LF}, \mathrm{HF}} \times 2 \times\left(\frac{27.5}{11}\right)^{2}$

where 27.5 and $11 \mathrm{~m}$ are the IRAM and APEX telescopes effective sizes, respectively, that is to say the portion of the primary mirrors that are optically conjugated to the cold pupils of the instruments (aperture stops).

Then we assumed a frequency window of $\Delta v=115 \mathrm{GHz}$, making the assumption that the two arrays cover the frequency range of $195-310 \mathrm{GHz}(\mathrm{HF})$ and $130-270 \mathrm{GHz}(\mathrm{LF})$, with a notch filter removing $25 \mathrm{GHz}$ of the low-frequency bandpass (around $183 \mathrm{GHz}$ ). Finally, we also had to take the decrease in transmission into account due to the increased optics complexity of CONCERTO compared to NIKA2 (in particular FTS optics will have some transmission loss and additional loading), which we estimate to be $T=0.8$ for an unpolarised source. It is important to note that this is only an additional loss of transmission compared to NIKA2+P1 and not the overall transmission.

The sensitivity of CONCERTO as a dual-band photometer that is set when the optical path difference in the FTS is null is thus:

$\mathrm{NEFD}_{\text {phot }}^{\mathrm{LF}, \mathrm{HF}}=\mathrm{NEFD}^{\mathrm{LF}, \mathrm{HF}} \times \sqrt{\frac{\Delta v_{\mathrm{NIKA} 2}^{\mathrm{LF}, \mathrm{HF}}}{\Delta v}} \times \frac{1}{\sqrt{T}}$.

We have $\Delta v_{\mathrm{NIKA} 2}^{\mathrm{HF}}=48 \mathrm{GHz}$ and $\Delta v_{\mathrm{NIKA} 2}^{\mathrm{LH}}=39.2 \mathrm{GHz}$ (Perotto et al. 2020) and thus: 
Table 2. Key parameters for CONCERTO instrument.

\begin{tabular}{|c|c|c|c|c|c|c|}
\hline$v[\mathrm{GHz}]$ & 131 & 156 & 211 & 238 & 272 & 302 \\
\hline Redshift of the $[\mathrm{CII}]$ line & 13.5 & 11.2 & 8.0 & 7.0 & 6.0 & 5.3 \\
\hline Beam size $[\operatorname{arcsec}]$ & 52.4 & 44.0 & 32.5 & 28.8 & 25.2 & 22.7 \\
\hline Beam solid angle $\left[\times 10^{-8} \mathrm{sr}\right]$ & 7.30 & 5.15 & 2.81 & 2.21 & 1.70 & 1.37 \\
\hline Mapping speed $\left[\times 10^{-3} \mathrm{deg}^{2} /\left(\mathrm{MJy} \mathrm{sr}^{-1}\right)^{2} \mathrm{~h}^{-1}\right]$ & $42.8[19.0-76.0]$ & $19.8[9.0-35.9]$ & $3.4[1.8-7.1]$ & $1.7[0.9-3.7]$ & $0.8[0.5-1.9]$ & $0.5[0.3-1.2]$ \\
\hline $\begin{array}{l}\text { On sky map sensitivity } \sigma_{\text {array }} \\
{\left[\left(\mathrm{MJy} \mathrm{sr}^{-1}\right) \mathrm{s}^{1 / 2}\right]}\end{array}$ & $2.1[1.5-3.1]$ & $3.0[2.3-4.5]$ & $7.3[5.1-10.2]$ & $10.4[7.1-14.1]$ & $14.8[9.9-19.7]$ & $18.2[12.2-24.3]$ \\
\hline
\end{tabular}

Notes. Sensitivities and mapping speeds are given for one spectral element (with $\delta v=1.5 \mathrm{GHz}$ ), assuming $80 \%$ of valid KIDS, a precipitable water vapour of $2 \mathrm{~mm}$, and an elevation of 60 degrees.

$\mathrm{NEFD}_{\text {phot }}^{\mathrm{LF}}=81.6[61.2-122.4] \mathrm{mJy} \mathrm{s}^{1 / 2}$ and $\mathrm{NEFD}_{\text {phot }}^{\mathrm{HF}}=135.4$ [90.3-180.6] $\mathrm{mJy} \mathrm{s}^{1 / 2}$.

We note that due to the FTS in front of the cryostat, CONCERTO is a non-optimal instrument for imaging. However, the option of removing the first polariser for purely photometric campaigns could be studied. In that case, the sensitivity is expected to be two times better for each individual array (as we took a factor 2 of penalty for P1).

\subsection{Sensitivity in spectroscopy}

For the spectroscopic mode, we consider a fix value for spectral resolution of $\delta v=1.5 \mathrm{GHz}$. The number of spectral elements in the frequency range is $N_{\mathrm{se}}=\Delta v / \delta v$.

The sensitivity per spectral element (in $\mathrm{mJy} \mathrm{s}^{1 / 2}$ ) for a single spectrometer (we note that in our case, with an FTS, the number of pixel equals the number of spectrometers) is given by

$\mathrm{NEFD}_{\text {FTS }}=\mathrm{NEFD}_{\text {phot }} \times N_{\text {se }}$.

The beam area is computed assuming a Gaussian beam,

$\Omega_{\text {beam }}=2 \pi\left(\frac{\theta_{\text {beam }}}{2 \sqrt{2 \log 2}}\right)^{2}$,

with a FWHM that is determined by the Rayleigh criterion for a $D=11 \mathrm{~m}$ antenna (our illumination of the APEX $12 \mathrm{~m}$ antenna) at a given frequency, (which corresponds to a given redshift for the $[\mathrm{CII}]$ line),

$\theta_{\text {beam }}=1.22 \lambda_{\text {obs }} / D$.

We can then convert the sensitivity per spectral element from point-source (Eq. (5)) to diffuse emission (in $\mathrm{MJy} \mathrm{sr}^{-1} \mathrm{~s}^{1 / 2}$ ) following

$\mathrm{NEI}_{\mathrm{FTS}}=\mathrm{NEFD}_{\mathrm{FTS}} \times 10^{-9} / \Omega_{\text {beam }}$.

This is the noise equivalent intensity, on sky, per KIDS, per spectral bin (taken as $\delta v=1.5 \mathrm{GHz}$ ).

We can finally compute the mapping speed MS (per spectral element) following,

$\mathrm{MS}=\mathrm{FOV} / \mathrm{NEI}_{\mathrm{FTS}}^{2}$,

where FOV is the field of view area (with a diameter of 20 arcmin). The numbers are given in Table 2. We note that we ignored the frequency overlap between the two arrays (and thus a gain of $\sim \sqrt{2}$ on the sensitivity in the frequency overlap region). We considered $\mathrm{NEFD}_{\mathrm{DB}}^{\mathrm{LF}}$ for $v \leq 150 \mathrm{GHz}, \mathrm{NEFD}_{\mathrm{DB}}^{\mathrm{HF}}$ for $v \geq 260 \mathrm{GHz}$, and a linear interpolation between the two NEFDs for $150<v<260 \mathrm{GHz}$.
In Table 2, we also provide the sensitivity for the whole array, per spectral element, which is:

$\sigma_{\text {array }}=\mathrm{NEI}_{\mathrm{FTS}} / \sqrt{N_{\mathrm{KIDS}}}$,

where $N_{\text {KIDS }}$ is the number of pixels (KIDS) of each array; we use 1720 KIDS, which correspond to $80 \%$ of valid KIDS in each array. This would be the sensitivity of each voxel ${ }^{9}$ of large maps, assuming a RA-Dec (or AZ-EL) raster scan-like scanning strategy and also assuming that pixel sizes of the map are equal to beam sizes. Each voxel of the observed map would then be observed by each KIDS. We checked these numbers using a scanning strategy similar to NIKA2 raster scans, with three interferograms per beam.

\section{Low spectral-resolution spectroscopic surveys}

CONCERTO will offer generic access to a large FoV and a lowfrequency resolution spectroscopic instrument. This opening of 3D large-scale surveys is the next step after the broad-band photometric experiments, either from the ground (e.g. LABOCA, SCUBA2, NIKA2) or from space (e.g. Herschel and Planck). The first scientific aim of CONCERTO is to map, in three dimensions, the fluctuations of the [CII] line intensity in the reionisation and post-reionisation epoch $(z \geq 5.3)$. This technique, known as intensity mapping, will measure the clustering of [CII] emissivity and allow for questions to be answered on how and when galaxies and quasars formed and on the history and topology of reionisation. Even if [CII] intensity mapping has been the basis of instrument definition, we extended the instrument capabilities to make CONCERTO a multi-purpose instrument (e.g. extending the frequency range down to $130 \mathrm{GHz}$ for observations of galaxies clusters). Thus we expect CONCERTO to bring a significant contribution in a number of areas, including the study of galaxy clusters (via the thermal and kinetic SZ effect), the follow-up of cosmological deep surveys, the observation of local and intermediate-redshift galaxies, and the study of Galactic star-forming clouds. In this section we provide a forecast on the expected signal-to-noise ratio $(\mathrm{S} / \mathrm{N})$ that can be obtained on the $[\mathrm{CII}]$-emission power spectrum (Sect. 5.1). In addition, we give some predictions for observing the SZ signal of galaxy clusters (Sect. 5.2).

\section{1. [CII] intensity mapping with CONCERTO}

The [CII] line is one of the brightest emission lines in the spectra of galaxies. It is an excellent coolant for neutral gas in

9 A voxel represents a value on a regular grid in three-dimensional space. 
photo-dominated regions and an extinction-free tracer of star formation at high $z$. Being redshifted into the sub-millimetre and millimetre atmospheric windows for $z>4.5$, it has become one of the most popular lines at high $z$. Pointing on known objects, with, for example, ALMA, NOEMA, and APEX/FLASH, [CII] is now detected in a large number of galaxies at high $z(>150$ at $z>4.5$, with a large contribution from the ALMA ALPINE survey, e.g. Bethermin et al. 2020). These types of observations are a tremendous step forward but we also need to look at the overall population, that is, observe large volume and unbiased surveys. First observations with ground-based interferometers, for example, with ALMA ('ALMA Spectroscopic Survey in the Hubble Ultra Deep Field' large programme - ASPECS, Walter et al. 2016) or JVLA (CO Luminosity Density at High Redshift survey - COLDz, Riechers et al. 2019) offer a three-dimensional view of the molecular gas content of galaxies. The covered areas are about 5-60 square arcmin.

Intensity mapping complements these efforts beautifully (e.g Kovetz et al. 2019), providing an unbiased view of the distribution of the gas that is difficult to assemble from targeted measurements of individual galaxies, and probing cosmological volumes, with maps on several-degree scale and large frequency (and thus redshift) coverage. Intensity mapping exploits the confusion-limited regime and measures the integrated light emission from all sources, including unresolved faint galaxies.

We will conduct with CONCERTO a major survey of about one square degree with $1200 \mathrm{~h}$ of APEX telescope time. The survey will provide a data cube in which intensity is mapped as a function of sky position and redshift. Our main target is the [CII] line emission at $z \geq 5.3$. But CONCERTO will also observe the $\mathrm{CO}$ intensity fluctuations arising from $0.3<z<2$ galaxies, giving the spatial distribution and abundance of molecular gas over a broad range of cosmic time. The 3D fluctuations will be studied in Fourier space with the power spectrum.

To compute the expected $\mathrm{S} / \mathrm{N}$ on the $[\mathrm{CII}]$ power spectrum at high $z$, we used the [CII] model presented in Serra et al. (2016). Using measurements of the cosmic infrared background (CIB) angular power spectra from Herschel/SPIRE together with star formation rate density (SFRD) measurements, they constrain the galaxy FIR luminosity as a function of dark-matter halo mass at all relevant redshifts in the halo model framework. By using scaling relations from Spinoglio et al. (2012) to link the intensity of emission lines to the galaxy infrared luminosity, they computed 3D emission line power spectra for all relevant lines, including [CII]. They computed the expected $\mathrm{S} / \mathrm{N}$ of cross-power spectra between $[\mathrm{CII}]$ and other emission lines, which will constrain the mean amplitude of each signal and thereby help to gain insight into the mean properties of the ISM of high- $z$ galaxies. It is important to note that in their paper, they use for CONCERTO a constant sensitivity for all redshifts of $\sigma_{\text {array }}=155 \mathrm{mJy} \mathrm{s}^{1 / 2}$, while we have here $\sigma_{\text {array }}=[156,206,230,250,250] \mathrm{mJy} \mathrm{s}^{1 / 2}$ at $z=[11.2,8.0,7.0,6.0,5.3]$.

We followed Gong et al. (2012) to compute uncertainties on the power spectra. The observing time per map voxel, considering one pixel equals one beam, is given by

$t_{\text {voxel }}=t_{\text {survey }} \frac{\Omega_{\text {beam }} N_{\mathrm{KIDS}}}{A}$,

with A the survey area, $t_{\text {survey }}$ the on-sky survey time (i.e. $1200 \times 0.7=840 \mathrm{~h}$ in our case, considering $30 \%$ of overheads), $\Omega_{\text {beam }}$ the solid angle of the beam (Table 2), and $N_{\mathrm{KIDS}}$, the number of pixels (we consider $80 \%$ of valid KIDS, thus $N_{\text {KIDS }}=1720$ ).
Assuming a spherically averaged power spectrum measurement and a directionally independent on sky sensitivity, the variance of the power spectrum is:

$\operatorname{var}\left[\bar{P}_{\mathrm{CII}}(k)\right]=\frac{\left[P_{\mathrm{CII}}(k)+\bar{P}_{\mathrm{CII}}^{N}(k)\right]^{2}}{N_{\mathrm{m}}(k, z)}$,

where $N_{\mathrm{m}}(k, z)$ is the number of modes that leads to the power spectrum measurement at each $k$ and

$\bar{P}_{\text {CII }}^{N}(k)=V_{\text {voxel }} \frac{\sigma_{\text {voxel }}^{2}}{t_{\text {voxel }}}$,

with $V_{\text {voxel }}$ the volume surveyed by each voxel. In the case of CONCERTO, each KIDS gets a spectrum, and considering one KID per beam, we have $\sigma_{\text {voxel }}=\sigma_{\text {array }}$.

The number of modes at each $k$ is given by

$N_{\mathrm{m}}(k, z)=2 \pi k^{2} \Delta k \frac{V_{\mathrm{s}}(z)}{(2 \pi)^{3}}$,

where $\Delta k$ is the Fourier bin size and $V_{\mathrm{s}}(z)$ is the survey volume, expressed as

$V_{\mathrm{s}}(z)=\chi(z)^{2} y_{\mathrm{CII}} B_{v} A$,

with

$y_{\mathrm{CII}}(z)=\lambda_{\mathrm{CII}}(1+z)^{2} / H(z)$,

being the factor to convert the frequency intervals to the comoving distance at the wavelength $\lambda_{\mathrm{CII}}$ (rest frame wavelength of the [CII] line). In Eq. (15), $B_{v}$ is the bandwidth considered for the measurement. The volume surveyed by each voxel ( $V_{\text {voxel }}$ in Eq. (13)) is

$V_{\text {voxel }}=\chi(z)^{2} y_{\text {CII }}(z) \Omega_{\text {beam }} \delta_{v}$.

We consider measurements spanning a redshift range of $\Delta z \sim 0.6$, which corresponds to a frequency range of $B_{v} \sim$ $20 \mathrm{GHz}$ at $z=6.1$ for the [CII] line. We note that Gong et al. (2012) give useful relations for computing $V_{\mathrm{s}}$ and $V_{\text {voxel }}$ for the [CII] line.

Table 3 gives the numbers derived from the above computations and Fig. 16 shows the predicted [CII] power spectrum with its error bars. Because these types of predictions are very uncertain, we assumed two extreme models, giving respectively low and high SFRD at $z>3$ (see Fig. 3 of Lagache et al. 2018). Low SFRD is the pessimistic prediction as it corresponds to the lowest UV-driven SFRD; high SFRD is the optimistic prediction as it corresponds to the CIB-driven SFRD derived from the halo modelling of Planck CIB measurements (Planck Collaboration XXX 2014).

Covering an area of 1.4 square degrees, our survey will provide the first measurements of the [CII] power spectrum up to $z \sim 7$, considering the mean SFRD and average sensitivity estimate, and up to $z \sim 8$ in the best case for the sensitivity estimate. We note that the low SFRD case is unlikely as it gives low shot-noise levels for the CIB, which are not compatible with the Planck and Herschel measurements nor with current SFRD measurements at high- $z$ based on $[\mathrm{CII}]$ or far-infrared measurements. Moreover, on top of the exact level of the SFRD at high $z$, the relation used to convert SFR to [CII] luminosity is another capital ingredient of this type of model. Serra et al. (2016) used the relation from Spinoglio et al. (2012), which provides, for a given SFR, a [CII] luminosity that is six times lower than that obtained with the [CII]-SFR relations of Lagache et al. (2018) 
Table 3. $\mathrm{S} / \mathrm{N}$ on the $[\mathrm{CII}]$ power spectrum.

\begin{tabular}{lcccc}
\hline \hline Redshift $z$ & 5.5 & 6.2 & 7 & 8 \\
\hline S/N $P^{\text {CII }}$ mean SFRD & $23[14-44]$ & $12[6.9-24]$ & $5.7[3.1-12]$ & $2.0[1.0-4.1]$ \\
S/N $P^{\text {CII }}$ low SFRD & $4.5[2.6-9.8]$ & $1.9[1.1-4.3]$ & $0.78[0.48-1.7]$ & $0.23[0.12-0.48]$ \\
S/N $P^{\text {CII }}$ high SFRD & $79[57-112]$ & $55[36-87]$ & $34[21-60]$ & $16[8.5-29]$ \\
\hline
\end{tabular}

Notes. $P^{\mathrm{CII}}$ was computed for $\Delta z=0.6$ and given for $k=[0.1,1] h \mathrm{Mpc}^{-1}$. See the caption of Fig. 16 for more details. Numbers in brackets reflect the range of sensitivities (as given in Table 2).

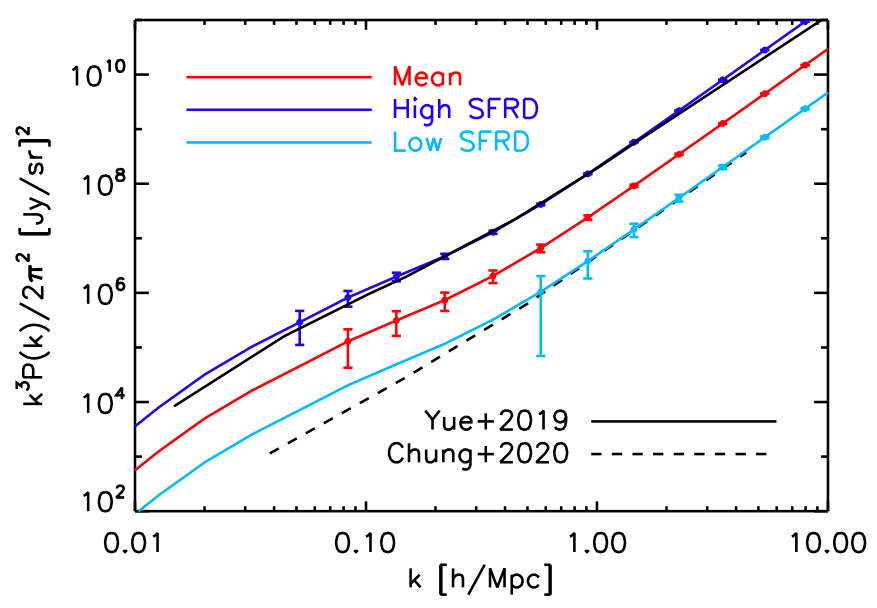

Fig. 16. Predicted $[\mathrm{CII}]$ power spectrum at $z=6$. Three cases are shown, corresponding to three scenarios of SFRD at high $z$ (see Lagache et al. 2018): high SFRD (dark blue), low SFRD (light blue), and the geometrical mean of the two (red). Only points with $S / N>1$ are shown. $\mathrm{S} / \mathrm{Ns}$ were computed considering $A=1.4$ square degrees, $t_{\text {survey }}=840 \mathrm{~h}$, which corresponds to a total observation time of 1200 hours taking the overheads into account, and sensitivities as estimated in Sect. 4. The [CII] power spectra were derived from the modelling of CIB power spectra (Serra et al. 2016), using a conversion from SFR to [CII] that conservatively underestimates the [CII] luminosity by a factor of 6 at $z=5$ compared to recent semi-analytical models (e.g. Lagache et al. 2018) or ALMA ALPINE measurements (Schaerer et al. 2020). Our estimates of [CII] power spectra are thus likely to be underestimated. The predicted [CII] power spectra from Yue \& Ferrara 2019, using the local SFR-[CII] relation (black line) and Chung et al. 2020 (dashed black line) are also shown.

or observed with the ALMA ALPINE survey (Schaerer et al. 2020). Our power spectra are thus very likely to be underestimated. In terms of point source sensitivities, our survey will reach $1 \sigma=[14.4,14.9,19.7,22.0,23.9,23.9] \mathrm{mJy}$, for a spectral element $\delta v=1.5 \mathrm{GHz}$, at $v=[131.0,156.0,211.0,238.0,272.0$, $302] \mathrm{GHz}$, respectively. Finally, one major difficulty in intensity mapping surveys is the problem of foregrounds. For [CII], the main foregrounds will be the contamination from emission lines from lower redshifts, in particular emission from $\mathrm{CO}$ rotation transitions (e.g. Yue et al. 2015). Silva et al. (2015) and Breysse et al. (2015) show that this contamination can be partially removed by masking out the brightest pixels in the survey or the low-redshift galaxies selected from other surveys. To that end, CONCERTO will highly benefit from the extensive visible-IR photometry and spectroscopy galaxy survey data that are available in the chosen field (i.e. the COSMOS field). In addition, one of the strengths of CONCERTO is in its wide frequency range: Several CO lines are simultaneously observed at the same redshift for all redshifts of $z>0.35$. The crosscorrelation between these lines will be a very powerful method to remove the contamination. This will be specifically addressed for CONCERTO in a future paper.

\subsection{Observing galaxy clusters with CONCERTO}

Clusters of galaxies are the largest gravitationally bound objects in the Universe and, as such, they are key to understanding the hierarchical large scale structure (Kravtsov \& Borgani 2012). The study of galaxy clusters and, in particular, their number as a function of mass and redshift allows us to constrain cosmological parameters (Allen et al. 2011). In the frequency range covered by CONCERTO, a cluster of galaxies will be mainly detected via the thermal and kinetic Sunyaev-Zel'dovitch (SZ) effect (Sunyaev \& Zel'dovich 1972; Sunyaev \& Zeldovich 1980). The thermal SZ (tSZ) effect (Sunyaev \& Zel'dovich 1972) refers to the interaction of the hot electrons in clusters with the $\mathrm{CMB}$ photons. It results in a distortion in the CMB spectrum at the position of the cluster. In the case of hot clusters, it will also be affected by relativistic corrections named rSZ (see Itoh \& Nozawa 2004, for details on their computation). The kinetic SZ (kSZ) (Sunyaev \& Zeldovich 1980) is a Doppler shift of the $\mathrm{CMB}$ photons induced by the proper motion of clusters of galaxies along the line-of-sight.

Observations of the tSZ effect have been successfully performed at high angular resolution using continuum cameras based on KIDs, such as NIKA and NIKA2 at the IRAM 30-m telescope (Adam et al. 2014, 2015, 2016, 2017a; Ruppin et al. 2017, 2018). For the kSZ effect, which is also observed with, for example, NIKA2 (Adam et al. 2017b), a multi-wavelength spectrometer such as CONCERTO would be a unique tool to separate the tSZ and kSZ as well as the different foreground components (CIB, CMB, and the Galactic emission) and to extract information about the cluster physics. Indeed, with sufficiently precise spectroscopy measurements (Birkinshaw 1999), we can measure the cluster mass (from the tSZ effect), proper motion along the line-of-sight (from the kSZ), and temperature (from the relativistic corrections to the tSZ).

Particularly, considering the angular resolution and the mapping speed of CONCERTO, we expect to perform a precise estimate of the shape of the SZ spectrum for clusters of galaxies for redshifts between 0.2 and 0.8 . As an illustration, we present in Fig. 17 a simulation of a typical cluster that could be observed with CONCERTO. In this simulation, the mass of the cluster is equal to $10^{15} M_{\odot}$ and the cluster is located at a redshift of $z=0.4$. We assumed a universal pressure profile model from Arnaud et al. (2010) to compute the cluster's Compton parameter map. From left to right, we present the cluster's SED as expected to be measured by CONCERTO at different radial distances from the centre of the cluster. We considered six bands in frequency with typical bandwidths of 10-25 GHz. We explored both the sensitivity to the relativistic SZ effect (blue points), assuming a cluster temperature of $20 \mathrm{keV}$, and to the kinetic SZ (red points), assuming the cluster is moving towards the observer 

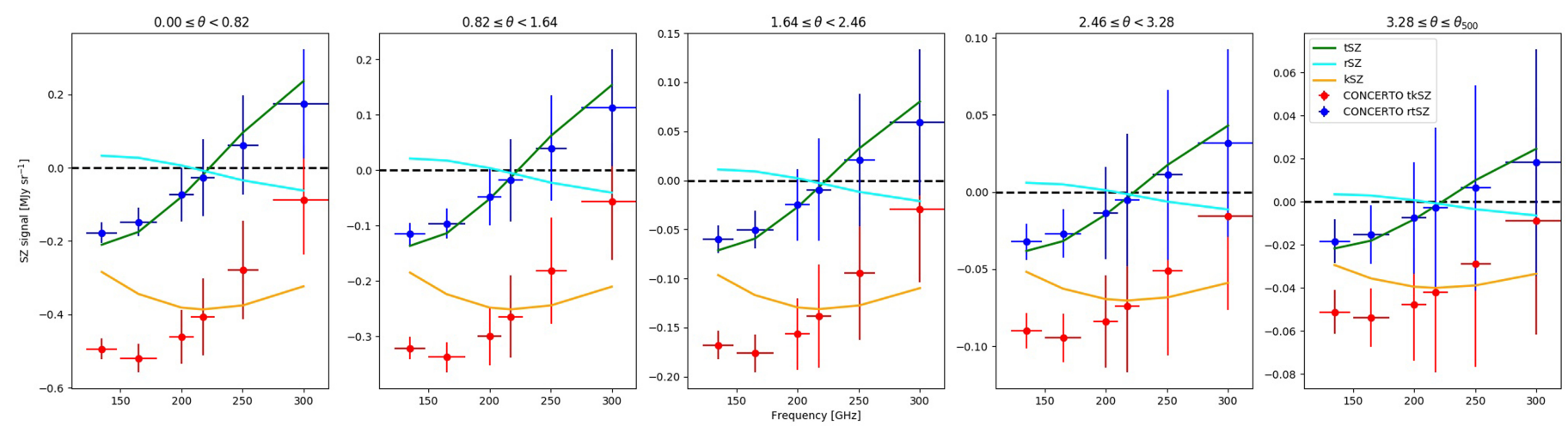

Fig. 17. Thermal (green), kinetic (orange), and relativistic (light blue) SZ effect for a simulated cluster at redshift $z=0.4$, with a mass of $10^{15} M_{\odot}$ and a temperature of $20 \mathrm{keV}$, which is moving at $1000 \mathrm{~km} \mathrm{~h}^{-1}$ towards the observer. From left to right, we present the measured CONCERTO SEDs for tSZ+kSZ and rSZ+tSZ, including uncertainties (red and blue dots, respectively) for different radial bins with respect to the centre of the cluster ( $\theta$ in arcmin) up to the cluster's characteristic radius, $\theta_{500}$, which is the radial angular distance at which the mean cluster density is 500 times the critical density at the cluster redshift.

with a velocity of $1000 \mathrm{~km} \mathrm{~h}^{-1}$. Uncertainties were computed from the sensitivity estimates given in Sect. 4, assuming a mapping area of $310 \mathrm{arcmin}^{2}$ and a total integration time of 30 hours. In the Fig. 17, we also show the individual tSZ, kSZ, and rSZ effect contributions.

We find that for reasonable observation times (tens of hours), CONCERTO would provide first spectral 2D mapping of the intracluster medium of high redshift clusters and should be able to measure cluster velocities via the $\mathrm{kSZ}$ effect. CONCERTO should also be able to detect the relativistic SZ effect and measure the cluster temperature. A more detailed mapping of the cluster temperature would require observation times of about hundreds of hours.

\section{Conclusions}

We have presented the design of the CONCERTO instrument, a novel spectrometer that is set to be installed on the APEX telescope. CONCERTO is based on the development of new arrays in the millimetre using kinetic inductance detectors. It will contain two arrays of 2152 KIDS, mounted in a dilution cryostat that has a base temperature of $0.1 \mathrm{~K}$. Spectra are obtained by a fast Martin-Puplett interferometer located in front of the cryostat. Frequency resolution can be up to $\delta_{v}=1 \mathrm{GHz}$. The technological choices leading to the final instrument design have been explained in detail. The characterisation of our current detectors is promising. Estimates of expected sensitivity are given, which are mostly based on the NIKA2 experience, that is, an instrument on sky that is subject to similar constraints as CONCERTO. The expected sensitivity, combined with the large field-of-view (20 arcmin diameter), will provide an unprecedented mapping speed for this kind of instrument.

CONCERTO will cover the frequency range of 130$310 \mathrm{GHz}$, allowing, in particular, the observation of the [CII]emission line at high redshift and the SZ signal from galaxy clusters. These two main science drivers for CONCERTO have been detailed in the paper. CONCERTO is particularly well-suited for these two objectives since (i) the [CII] line intensity mapping requires a combination of sensitivity, a large field-of-view, and a spectral resolution of $R \geq 100$ and (ii) the study of galaxies clusters via the SZ effect requires a multi-frequency analysis (to separate the different $\mathrm{SZ}$ signals as well as the SZ signals from CIB, CMB, and Galactic dust), the angular resolution of the 12 -m telescope, and $R \approx 10$.
CONCERTO is currently in an advanced stage of fabrication. The installation and technical commissioning at the APEX telescope is scheduled for the first semester of 2021. The commissioning, scientific verification, and observations are foreseen by the end of 2022 .

Acknowledgements. Besides the authors, the technicians and engineers more involved in the experimental setup development have been Maurice Grollier, Olivier Exshaw, Anne Gerardin, Gilles Pont, Guillaume Donnier-Valentin, Philippe Jeantet, Mathilde Heigeas, Christophe Vescovi, and Marc Marton. We acknowledge the crucial contributions of the whole Cryogenics and Electronics groups at Institut Néel and LPSC. The arrays described in this paper have been produced at the PTA Grenoble microfabrication facility. We warmly thank the support from the APEX staff for their help in CONCERTO pre-installations and design. The flexible pipes, in particular, have been routed under the competent coordination of Jorge Santana and Marcelo Navarro. We acknowledge support from the European Research Council (ERC) under the European Union's Horizon 2020 research and innovation programme (project CONCERTO, grant agreement No 788212) and from the Excellence Initiative of Aix-Marseille University-A*Midex, a French "Investissements d'Avenir" programme. GL warmly thanks Matt Bradford, Jamie Bock and Tzu-Ching Chang for insightful discussions on CONCERTO sensitivity computation and J.-G. Cuby for his help and support for the ERC proposal. We are grateful to our administrative staff in Grenoble and Marseille, in particular Patricia Poirier, Mathilde Berard, Lilia Todorov and Valérie Favre, and the Protisvalor team. We acknowledge the crucial help of the Institut Néel and MCBT Heads (Etienne Bustarret, Klaus Hasselbach, Thierry Fournier, Laurence Magaud) during the COVID-19 restriction period.

\section{References}

Adam, R., Comis, B., Macías-Pérez, J. F., et al. 2014, A\&A, 569, A66 Adam, R., Comis, B., Macías-Pérez, J.-F., et al. 2015, A\&A, 576, A12 Adam, R., Comis, B., Bartalucci, I., et al. 2016, A\&A, 586, A122 Adam, R., Arnaud, M., Bartalucci, I., et al. 2017a, A\&A, 606, A64 Adam, R., Bartalucci, I., Pratt, G. W., et al. 2017b, A\&A, 598, A115 Adam, R., Adane, A., Ade, P. A. R., et al. 2018, A\&A, 609, A115 Ade, P. A. R., Pisano, G., Tucker, C., \& Weaver, S. 2006, in A Review of Metal Mesh Filters, SPIE Conf. Ser., $627562750 U$

Allen, S. W., Evrard, A. E., \& Mantz, A. B. 2011, ARA\&A, 49, 409

Arnaud, M., Pratt, G. W., Piffaretti, R., et al. 2010, A\&A, 517, A92

Bethermin, M., Fudamoto, Y., Ginolfi, M., et al. 2020, A\&A, in press https://doi.org/10.1051/0004-6361/202037649

Birkinshaw, M. 1999, Phys. Rep., 310, 97

Bourrion, O., Vescovi, C., Bouly, J. L., et al. 2012, in SPIE Conf. Ser., 8452 Breysse, P. C., Kovetz, E. D., \& Kamionkowski, M. 2015, MNRAS, 452, 3408 Calvo, M., D’Addabbo, A., Monfardini, A., et al. 2014, J. Low Temp. Phys., 176, 518

Choi, S. K., Austermann, J., Basu, K., et al. 2020, J. Low Temp. Phys., 199, 1089

Chung, D. T., Viero, M. P., Church, S. E., \& Wechsler, R. H. 2020, ApJ, 892, 51 
Crites, A. T., Bock, J. J., Bradford, C. M., et al. 2014, in Proc. SPIE, SPIE Conf. Ser., 9153, 91531W

Doyle, S., Mauskopf, P., Zhang, J., et al. 2010, in A Review of the Lumped Element Kinetic Inductance Detector, SPIE Conf. Ser., 7741, 77410M

Fasano, A., Aguiar, M., Benoit, A., et al. 2020a, J. Low Temp. Phys., 199, 529

Fasano, A., Aguiar, M., Benoit, A., et al. 2010b, Eur. Phys. J. Web Conf., 228, 00010

Gong, Y., Cooray, A., Silva, M., et al. 2012, ApJ, 745, 49

Goupy, J., Adane, A., Benoit, A., et al. 2016, J. Low Temp. Phys., 184, 661

Griffin, M. J., Abergel, A., Abreu, A., et al. 2010, A\&A, 518, L3

Güsten, R., Nyman, L. Å., Schilke, P., et al. 2006, A\&A, 454, L13

Holland, W. S., Bintley, D., Chapin, E. L., et al. 2013, MNRAS, 430, 2513

Itoh, N., \& Nozawa, S. 2004, A\&A, 417, 827

Kovetz, E., Breysse, P. C., Lidz, A., et al. 2019, BAAS, 51, 101

Kravtsov, A. V., \& Borgani, S. 2012, ARA\&A, 50, 353

Kreysa, E., Gemuend, H. P., Gromke, J., et al. 1998, in Proc. SPIE, ed. T. G. Phillips, SPIE Conf. Ser., 3357, 319

Lagache, G. 2018, in Peering towards Cosmic Dawn, eds. V. Jelić, \& T. van der Hulst, IAU Symp., 333, 228

Lagache, G., Cousin, M., \& Chatzikos, M. 2018, A\&A, 609, A130

Lefèvre, C., Kramer, C., Neri, R., Berta, S., \& Schuster, K. 2020, in Eur. Phys. J. Web Conf., 228, 00014
Martin, D. H., \& Puplett, E. 1970, Infrared Phys., 10, 105

Mather, J. C., et al. 1999, ApJ, 512, 511

Monfardini, A., Benoit, A., Bideaud, A., et al. 2011, ApJS, 194, 24

Perotto, L., Ponthieu, N., Macías-Pérez, J. F., et al. 2020, A\&A, 637, A71

Planck Collaboration XXX. 2014, A\&A, 571, A30

Riechers, D. A., Pavesi, R., Sharon, C. E., et al. 2019, ApJ, 872, 7

Ruppin, F., Adam, R., Comis, B., et al. 2017, A\&A, 597, A110

Ruppin, F., Mayet, F., Pratt, G. W., et al. 2018, A\&A, 615, A112

Schaerer, D., Ginolfi, M., Bethermin, M., et al. 2020, A\&A, in press https://doi.org/10.1051/0004-6361/202037617

Schillaci, A., D’Alessandro, G., de Bernardis, P., et al. 2014, A\&A, 565, A125

Serra, P., Doré, O., \& Lagache, G. 2016, ApJ, 833, 153

Silva, M., Santos, M. G., Cooray, A., \& Gong, Y. 2015, ApJ, 806, 209

Siringo, G., Kreysa, E., Kovács, A., et al. 2009, A\&A, 497, 945

Spinoglio, L., Dasyra, K. M., Franceschini, A., et al. 2012, ApJ, 745, 171

Sunyaev, R. A., \& Zel'dovich, Y. B. 1972, Astrophys. Space Phys. Res., 4, 173

Sunyaev, R. A., \& Zeldovich, I. B. 1980, MNRAS, 190, 413

Walter, F., Decarli, R., Aravena, M., et al. 2016, ApJ, 833, 67

Yue, B., \& Ferrara, A. 2019, MNRAS, 490, 1928

Yue, B., Ferrara, A., Pallottini, A., Gallerani, S., \& Vallini, L. 2015, MNRAS, 450,3829 